\title{
Is the mitochondrion a promising drug target in trypanosomatids?
}

\author{
Yasmin Pedra-Rezende, Ana Cristina Souza Bombaça, Rubem Figueiredo Sadok Menna-Barreto/ ${ }^{+}$ \\ Fundação Oswaldo Cruz-Fiocruz, Instituto Oswaldo Cruz, Laboratório de Biologia Celular, Rio de Janeiro, RJ, Brasil
}

The trypanosomatids Trypanosoma brucei, Trypanosoma cruzi and Leishmania spp. are etiological agents of important neglected tropical diseases, affecting millions of people worldwide, and the drugs available for these diseases present several limitations. Novel efficient and nontoxic drugs are necessary as an alternative to the current chemotherapy. The unique mitochondrion of trypanosomatids and its peculiar features turn this organelle a potential drug target. Several phenotypic studies describe the damage in the parasite mitochondrial ultrastructure, but the molecular target is unknown. Few reports demonstrated the electron transport system (ETS) as a target due to the high similarities to mammalian orthologues, hence ETS is not a good candidate for drug intervention. On the other hand, antioxidant enzymes, such as trypanothione reductase, and an alternative oxidase (AOX) seem to be interesting targets; however no high active inhibitors were developed up to now. Finally, due to the remarkable differences to mammalian machinery, together with the high biological importance for the parasite survival, the mitochondrial import system stands out as a very promising target in trypanosomatids. Archaic translocase of the outer membrane (ATOM) and translocase of the inner membrane (TIM) complexes, which mediate both protein and tRNA import, composed by specific subunits of these parasites, could be excellent candidates, deserving studies focused on the development of specific drugs.

Key words: trypanosomatids - mitochondrion - oxidative stress - chemotherapy - mitochondrial protein import - bioenergetics

\section{The public health problem}

The human African trypanosomiasis, Chagas disease and leishmaniasis are neglected tropical diseases caused by the protozoa parasites Trypanosoma brucei, Trypanosoma cruzi and Leishmania spp respectively. These illnesses affect millions of people worldwide with high morbidity and mortality rates and lead to critical social-economic implications, since they persist under conditions of poverty and are concentrated in impoverished populations in the developing world.(1) Vaccines are not available, and the treatment of these diseases is based on drugs developed more than 50 years ago, especially due to the little or no prospect of financial gain, which discourages the search of new molecules by pharmaceutical industry. Additionally, current chemotherapy approaches have severe limitations, including high host toxicity, long-term treatments, and emergence of drugresistant strains..$^{(2,3)}$

\section{Potential drug targets in trypanosomatid mitochondrion and oxidative stress generation}

Parasites of Trypanosomatidae family exhibit the most typical eukaryotic organelles; however, some particular structures are also present, and have been pointed as potential targets for drug intervention. Unlike mammalian cells, trypanosomatids have a unique mitochondrion with several important metabolic peculiarities, which accredits this organelle as an excellent target for the development of novel therapeutics approaches.

doi: 10.1590/0074-02760210379

+ Corresponding author: rubemsadok@gmail.com / rubemb@ioc.fiocruz.br (D) https://orcid.org/ 0000-0002-1352-0641

Received 30 November 2021

Accepted 13 December 2021
(4,5) Such mitochondrion is also the most recurrent drug target described in mechanistic studies, with different classes of drugs inducing the organelle swelling, and impairing its matrix electron density and cristae ultrastructure (Fig. 1). ${ }^{(6,7,8,9)}$ Despite this promising scenario, mitochondrial phenotypes are commonly observed only by electron microscopy, with few reports assessing the real impact of these compounds in the organelle physiology.

In general, mitochondria are organelles essential to aerobic organisms and compartmentalise fundamental physiologic processes. A cytochrome-independent alternative oxidase (AOX) in trypanosomatids, mainly found in long slender bloodstream form of T. brucei, is a promising drug target since this molecule is absent in mammalian cells. ${ }^{(10)}$ This parasite form relies entirely on the catabolism of glucose, an abundant energy source in the bloodstream of mammalian host, using AOX as terminal oxidase enzyme in the aerobic respiratory pathway. Thus, the nicotinamide adenine dinucleotide (NADH) formed during glycolysis is re-oxidised by $\mathrm{O}_{2}$ in the parasites' mitochondrion in a process uncoupled to oxidative phosphorylation, and dependent of a mitochondrial membrane-associated glycerol-3-phosphate oxidase system, comprising a FAD-dependent glycerol3-phosphate dehydrogenase, ubiquinone and AOX.(11) Although, in bloodstream forms of T. brucei, the activity of mitochondrial complex IV (cytochrome oxidase) can be completely replaced by AOX, both molecules coexist in parasite's procyclic form, a life cycle stage marked by strong upregulation of mitochondrial energy metabolism and with components constituting a complete cytochrome-containing electron transport system (ETS). ${ }^{(12,13)}$ Additionally, the presence of AOX in other pathogenic trypanosomatids was not confirmed, despite the occurrence of complex IV-independent oxygen consumption has been described in T. cruzi and Leishmania (Leishmania) donovani. ${ }^{(14,15)}$ Altogether, these data raise the question whether an inhibitor of AOX also could be 
A

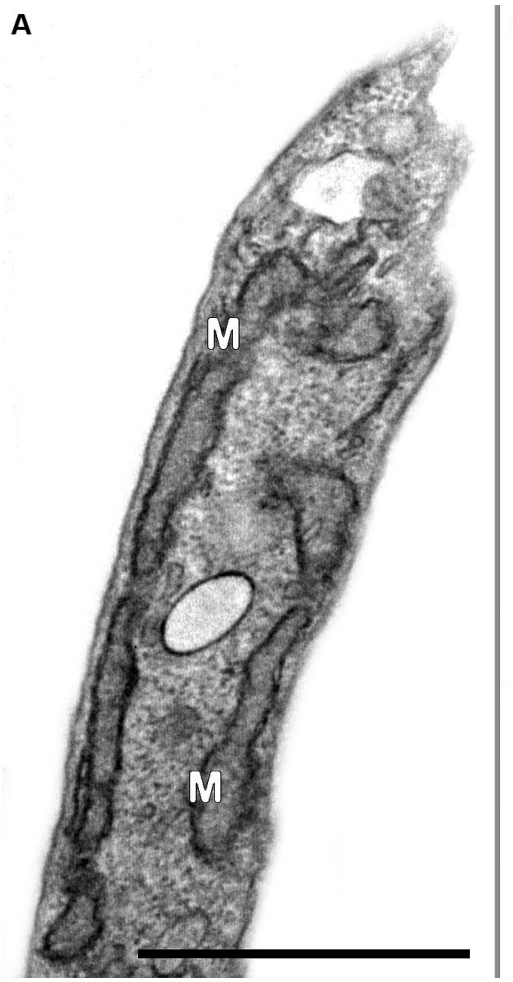

B

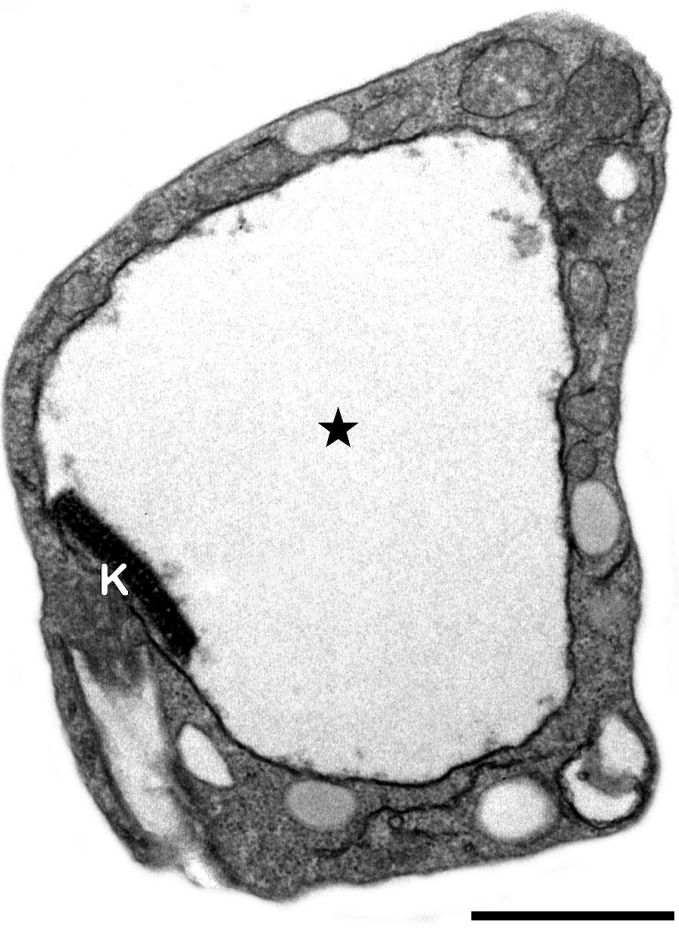

Fig. 1: mitochondrial swelling is the most recurrent ultrastructural phenotype detected in trypanosomatids after the treatment with drugs. (A) Untreated parasite presenting typical elongated morphology of the mitochondrion (M). (B) Treated parasite showing a remarkable dilation of the organelle with loss of the cristae and electron density of the matrix (star). K: kinetoplast; Bars $=1 \mu \mathrm{m}$.

a good candidate to the treatment of Chagas disease and leishmaniasis, since T. cruzi and Leishmania spp. have a more active mitochondrial metabolism, similarly to $T$. brucei procyclic form.

In addition to the pivotal role for energy production, mitochondrion also participates directly in the establishment of oxidative stress in eukaryotes, since the electron leakage from ETS leads to the partial reduction of oxygen and, consequently, the production of reactive oxygen species (ROS). ${ }^{(16)}$ In contrast to mammals, complex I (NADH-ubiquinone oxidoreductase) of trypanosomatids present low ROS production, which is directly associated with its truncated structure and low activity. ${ }^{(17,18,19)}$ Thus, the energy production in these parasites is fully dependent of complex II (succinate-ubiquinone oxidoreductase), and the succinate oxidation leads to electron transfer to the complex III (ubiquinolcytochrome c oxidoreductase) via ubiquinone. ${ }^{(19,20)}$ Although complexes II and IV are not common electron leakage sites, their inhibition by thenoyltrifluoroacetone (complex II inhibitor) or potassium cyanide (complex IV inhibitor) increases the ROS detection in trypanosomatids, as a consequence of electron flow interruption through ETS. ${ }^{(21,22,23)}$ Similar to mammalian cells, complex III and ubiquinone Q cycle are the major producers of ROS in these parasites. Such production occurs when ubiquinone remains for longer periods of time in its partially reduced form, due to the transference of only one electron from the complexes and formation of semiquinone, an intermediate extremely reactive and that can partially reduce other molecules present at the site, such as $\mathrm{O}_{2} \cdot{ }^{(24,25)}$ In $T$. cruzi and $L$. (L.) donovani, the treatment with antimycin A (complex III inhibitor) increases ROS production in parasite stages found in the insect. ${ }^{(21,22)}$

Although basal production of ROS is crucial for cell physiology, and fluctuations in their levels can occur in response to certain stimuli, high concentrations of these molecules induce oxidative stress, being necessary the removal in order to avoid toxicity. ${ }^{(26,27)}$ It has been extensively described that chemical characteristics can confer high redox potential to some classes of drugs, resulting in ROS production during the treatment. ${ }^{(6,28,29,30,31,32,33)}$ The first mechanistic studies evaluating the effects of quinones in trypanosomatids were performed in the late 1970's. In T. cruzi, epimastigotes treated with $\beta$-lapachone had increased mitochondrial ROS production, which was inhibited by the addition of the antioxidant enzyme superoxide dismutase. In this case, the generation of ROS was related to reduced respiratory rates and mitochondrial swelling in treated parasites. ${ }^{(34,35,36)}$

Previous studies of our group contributed to the elucidation of the trypanocidal mechanisms of $\beta$-lapachone derivatives. The reaction of this naphthoquinone with different aldehydes, in the presence of ammonium acetate, led to imidazole ring insertion, generating several naphthoimidazoles. N1, N2 and N3 (Fig. 2) were the most promising derivatives, presenting activity up to 18 fold higher on T. cruzi infective form than the reference drug. ${ }^{(37,38,39)}$ Despite the impairment of several cellular processes by these naphthoimidazoles, the most promi- 


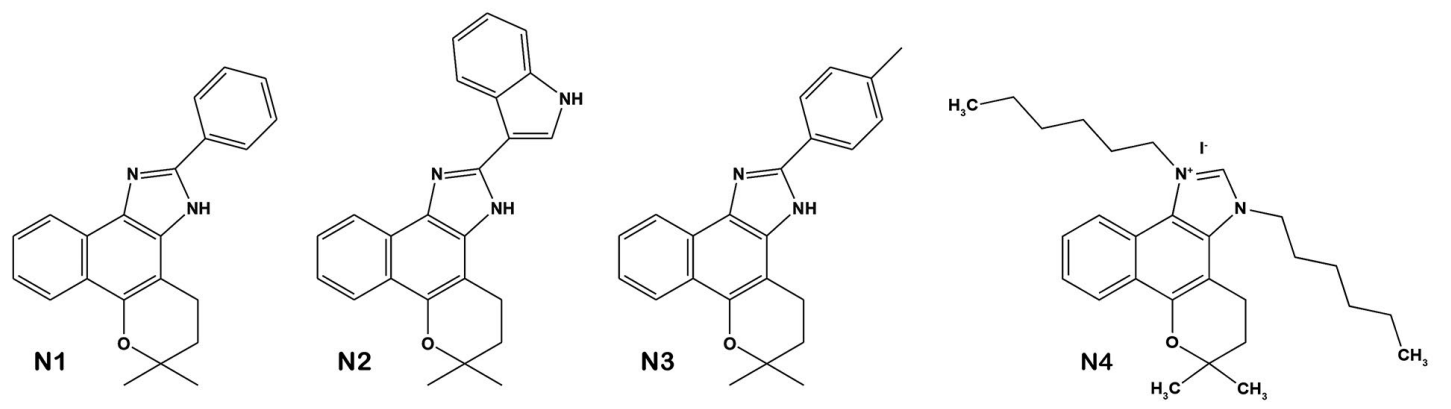

Fig. 2: chemical structures of naphthoimidazoles N1, N2, N3 and N4.

nent effect was observed in the protozoa mitochondrion. Ultrastructural analysis pointed to this organelle swelling in epimastigotes and bloodstream trypomastigotes, a significant reduction of mitochondrial membrane potential $(\Delta \Psi \mathrm{m})$ as well as in the activity of complex III. $(40,41)$ Corroborating these findings, in 2019, our study showed that these three derivatives compromised mitochondrion metabolism, disrupting $\mathrm{O}_{2}$ consumption and complexes II-III and IV activities. In addition, ROS were directly associated with the trypanocidal effect, N2 and N3 decreasing the electron flux through ETS and increasing the mitochondrial ROS production. In contrast, ROS production by $\mathrm{N} 1$ did not result from mitochondrial damage, so it may be a consequence of the antioxidant system inhibition. ${ }^{(32)}$ More recently, the correlation between mitochondrial ROS production and trypanocidal effect of the naphthoimidazole N4 (Fig. 2) was also demonstrated. The activity of N4 involves elevated ROS production and decreased activity of complex II-III in the first hours of treatment ( 2 and $4 \mathrm{~h}$ ); apart from the morphological and functional impairment of this organelle in longer times $(24 \mathrm{~h}) .^{(33)}$ In 2009 , our group also evidenced the effect of naphthofuranquinones in the mitochondrial metabolism of T. cruzi stages. These compounds exhibited powerful effects on the protozoa mitochondrion, which appeared drastically swollen and with a washed-out matrix phenotype, in addition to significant reduction of complex I-III activity and succinate-induced $\mathrm{O}_{2}$ consumption. It was suggested that naphthofuranquinones could interfere with electron flow at the inner mitochondrial membrane, deviating electrons from the total ubiquinone pool, resulting in semiquinone formation and oxidative stress. ${ }^{(6)}$ In addition, the treatment of T. cruzi with 1,2-naphthoquinone and 9,10-phenanthrenquinone led to superoxide anion and hydroxyl production, corroborating redox stress as the main mechanism of quinones. ${ }^{(42)}$

Quinones also had their mechanism of action assessed in other pathogenic trypanosomatids. ${ }^{(43)}$ In $L$. (L.) donovani, a benzophenone-derived bisphosphonium salt targets complex II and causes dramatic mitochondrial injury, including organelle swelling, $\Delta \Psi \mathrm{m}$ and $\mathrm{O}_{2}$ consumption decrease, as well as prominent impairment of cytoplasmic ATP levels ${ }^{7}$. Tafenoquine inhibits complex III, increases mitochondrial ROS production and elevates intracellular $\mathrm{Ca}^{2+}$ levels, inducing this organelle depolarisation in promastigotes forms of Leishmania spp. ${ }^{(30)} \mathrm{Sim}-$ ilar findings were observed in $L$. (L.) donovani treated with clerodane diterpene, leading to cytoplasmic cytochrome c release and disruption of ATP production. ${ }^{(31)}$

For many years the idea that trypanosomatids were more sensitive to oxidative stress, due to the lacking of classical antioxidant defenses such as catalase and glutathione peroxidase, led to the search for novel ROSproducing compounds in these parasites. ${ }^{(44,45)}$ However, this hypothesis has been proved incorrect, since the parasites' antioxidant system is extremely efficient fighting against reactive species derived from pharmacological intervention and/or produced in the host environment. Differently from mammalian cells, trypanosomatids possess a specific antioxidant system, based on glutathione analogue trypanothione $\left[\mathrm{T}(\mathrm{SH})_{2}\right] \cdot{ }^{(46)} \mathrm{T}(\mathrm{SH})_{2}$ is the major redox reactive metabolite in these parasites, and participates in a wide range of enzymatic and non-enzymatic reactions, such as peroxide detoxification. ${ }^{(47,48)}$ Accordingly, $\mathrm{T}(\mathrm{SH})$, plays a key role in redox homeostasis in all trypanosomatids, supporting the infectivity and survival in the hosts, while the disruption of its metabolism could increase parasites' susceptibility to oxidative stress. T(SH) $)_{2}$ pathway offers exceptional chances for the development of anti-trypanosomatids' strategies, which is desirable to decrease drug development costs and to treat many related diseases. ${ }^{(49)}$ Despite the major efforts in this field, focusing on metabolic enzymes involved in $\mathrm{T}(\mathrm{SH})_{2}$ synthesis and regeneration, no promising inhibitor has been found up to now. It is interesting to note that trypanosomatids can survive with reductions of up to $90 \%$ in trypanothione reductase activity and $\mathrm{T}(\mathrm{SH})_{2}$ levels, ${ }^{(50,51)}$ making hard the development of drugs able to inhibit this pathway.

\section{The mitochondrial protein import system is a promising drug target?}

The unique mitochondrion of trypanosomatids is extremely dynamic, adapting its morphology and content in response to environmental conditions found in host system. The huge majority of mitochondrial proteins are encoded by nuclear genome, depending on translocase of the outer membrane (TOM), translocase of the inner membrane (TIM), sorting and assembly machinery (SAM) and oxidase assembly (OXA) complexes for the import of polypeptide sequences into the organelle (Table). The mitochondrial outer membrane (MOM) protein Tom40 is the usual entry of polypeptide se- 
TABLE

Components of the mitochondrial protein import complexes in Trypanosoma brucei

\begin{tabular}{|c|c|c|c|c|}
\hline Complexes & Subunits & Depletion effects & Yeast ortholog & References \\
\hline \multirow{7}{*}{ ATOM } & ATOM40 & Reduction in levels of ATOM14, ATOM11, ATOM46, and ATOM69 & Tom40 & $(52,53,82,83)$ \\
\hline & ATOM14 & Impairment of protein and tRNA import & Tom 22 & $(53,83)$ \\
\hline & ATOM46 & not described & - & (53) \\
\hline & ATOM69 & Accumulation of cytosolic precursor proteins; parasite growth arrest & - & (53) \\
\hline & ATOM11 & Inhibition of tRNA import & - & $(53,83)$ \\
\hline & ATOM12 & Inhibition of tRNA import & - & (53) \\
\hline & ATOM19 & $\begin{array}{l}\text { Inhibition of mitochondrial protein import, } \Delta \Psi \mathrm{m} \text { and kDNA loss, } \\
\text { decline in } \mathrm{O}_{2} \text { uptake and parasite growth arrest }\end{array}$ & - & $(84,85)$ \\
\hline \multirow{2}{*}{ SAM } & Sam50 & not described & Sam50 & (56) \\
\hline & Sam35 & not described & Sam35 & (57) \\
\hline \multirow{2}{*}{ OTHERS } & pATOM36 & $\begin{array}{l}\text { Alteration in MOM protein composition, kDNA loss } \\
\text { and increased distance basal body - MOM }\end{array}$ & - & $(58,62)$ \\
\hline & Erv1 & $\begin{array}{l}\text { Reduction in abundance of small TIMs } \\
\text { and cysteine-rich substrates in MIS }\end{array}$ & Erv1 & $(70,71)$ \\
\hline \multirow{9}{*}{ TIM } & TbTim17 & $\begin{array}{l}\text { Inhibition of the mitochondrial import of proteins and the newly } \\
\text { synthesised tRNA; impairment of the mitochondrial import of } \\
\text { cytochrome oxidase subunit IV and decreased } \Delta \Psi \mathrm{m}\end{array}$ & $\operatorname{Tim} 17 / 22 / 23$ & $(54,75,76,86)$ \\
\hline & TbTim62 & $\begin{array}{l}\text { Inhibition of import of mitochondrial proteins in vitro; reduction in } \\
\text { mitochondrial protein content in vivo }\end{array}$ & - & $(54,75)$ \\
\hline & ACAD & not described & - & $(54,75)$ \\
\hline & TbTim42 & not described & - & (54) \\
\hline & TimRhom I & Impairment of cytochrome oxidase subunit 4 import & - & $(54,87)$ \\
\hline & TimRhom II & Impairment of cytochrome oxidase subunit 4 import & - & $(54,87)$ \\
\hline & TbTim50 & $\begin{array}{l}\text { kDNA overreplication, } \Delta \Psi \mathrm{m} \text { decrease and cell growth in the } \\
\text { bloodstream form of } T \text {. brucei }\end{array}$ & - & $(80,81)$ \\
\hline & TbTim47 & Inhibit in vitro import of mitochondrial proteins & - & (75) \\
\hline & TbTim54 & $\begin{array}{l}\text { Inhibit in vitro import of mitochondrial proteins; impacts negatively } \\
\text { the protein content in vivo }\end{array}$ & - & (75) \\
\hline \multirow{5}{*}{ PAM } & mHsp70 & Impairs mitochondrial import of proteins and tRNA & mHsp70 & $(75,76)$ \\
\hline & Mge1 & not described & Mge1 & (88) \\
\hline & TbPam16 & Parasite growth arrest & - & (79) \\
\hline & TbPam18 & Parasite growth arrest & - & (79) \\
\hline & $\operatorname{TbPam} 27$ & $\begin{array}{l}\text { Parasite growth arrest; and accumulation of cytochrome } \\
\text { oxidase subunit } 4\end{array}$ & - & (79) \\
\hline \multirow{6}{*}{ Small Tims } & Tim9 & Parasite growth arrest and reduced levels of TbTim 17 & Tim9 & $(54,64,68)$ \\
\hline & Tim10 & Parasite growth arrest and reduced levels of TbTim 17 & Tim10 & $(54,64,68)$ \\
\hline & $\operatorname{Tim} 8 / 13$ & Parasite growth arrest and reduced levels of TbTim 17 & $\operatorname{Tim} 8 / 13$ & $(54,64,66,68)$ \\
\hline & Tim11 & not described & - & $(54,66)$ \\
\hline & $\operatorname{Tim} 12$ & not described & - & $(54,66)$ \\
\hline & $\operatorname{Tim} 13$ & not described & - & (54) \\
\hline
\end{tabular}

quences in the organelle of most eukaryotes; however, in trypanosomatids, Tom40 is absent and is replaced by a different import channel, termed ATOM (archaic translocase of the outer mitochondrial). ${ }^{(52)}$ This complex was first described in T. brucei and is formed by seven subunits, two of them (ATOM40 and ATOM14) with some homology to Tom40 and Tom 22 present in yeast. (53) Although the other subunits (ATOM69, ATOM46, ATOM19, ATOM12, and ATOM11) are exclusive of trypanosomatids, and have evolved independently of any subunit previously described in the TOM complex, ${ }^{(53,54)}$ there are functional similarities between mitochondrial 
import systems of these parasites and other eukaryotic cells. Except for ATOM46, all subunits are essential for the viability and mitochondrial protein import in trypanosomatids. ${ }^{(55)}$ SAM complex in MOM was also described in T. brucei and its core subunit Sam50 is highly conserved. As in other eukaryotes, trypanosome Sam50 mediates the biogenesis of $\beta$-barrel membrane proteins into the outer membrane. ${ }^{(56)}$ An orthologue of Sam35, a component of SAM complex in yeast, has been detected in the MOM of trypanosomes; however, whether it forms a complex with Sam50 is presently unknown. . 55,57$)^{-5}$

Trypanosomatids also possess a specific protein, named pATOM36 (peripheral ATOM36), which is associated with insertion of some proteins in mitochondrial membrane and mediates the assembly of MOM protein complexes. pATOM36 knockdown affects MOM protein composition by molecules that have classical $\alpha$-helical transmembrane domains, including six subunits of the ATOM complex..$^{(58,59,60)}$ Despite the similar function with the mitochondrial inner-membrane import machinery (MIM) complex of yeast, pATOM36 has different molecular weight and topology, and does not show any sequence similarity to Mim1 or Mim2 (subunits of MIM complex). Interestingly, the expression of pATOM36 in a MIM-depleted strain rescued the growth of the cells and to the assembly of TOM complex in yeast; in the same way, the expression of Mim1 and Mim2 was not enough to assembly of ATOM after T. brucei pATOM36 knockdown. Altogether, these data suggest that pATOM36 and MIM complexes are functional analogues, and that neither the trypanosomal molecule nor Mim1/Mim2 needs a specific partner protein to exert their function. ${ }^{(61)}$ pATOM36 also has a second function unrelated to mitochondrial protein import. It has been pointed to the specific localisation of this molecule at the tripartite attachment complex (TAC), a trypanosomatid structure that physically links mitochondrial genome (kDNA) to the basal body of the parasite. ${ }^{(59)}$ Thus, $\mathrm{TAC}$ function is to guaranteeing the perfect segregation of the replicated kDNA to both daughter cells after mitosis. The knockdown of T. brucei pATOM36 also resulted in the loss of kDNA and increased the distance between basal body and MOM, indicating that this molecule is essential to DNA inheritance..$^{(62,63)}$

Small TIM (translocase of the inner mitochondrial membranes) proteins are a highly conserved family found in all eukaryotes. In T. brucei, six different small TIMs proteins with cysteine-rich motifs were described (TbTim9, TbTim10, TbTim11, TbTim12, TbTim13, and TbTim8/13); however, sequence comparisons do not allow the assignment of the trypanosomal TIMs to their counterparts in yeast and mammals. ${ }^{(55,64,65)}$ It has been determinated that all of these small TbTims are essential for $T$. brucei cell growth and are found tightly associated with TbTim17. Depletion of any of these severely hampers the stability of the TbTIM17 complex. ${ }^{(65,66,67,68)}$ The import of proteins in the mitochondrial intermembrane space (MIS) is mediated by MIA (mitochondrial intermembrane space assembly) system, consisting by Mia40 and Erv1 in yeast. Mia40 acts as receptor of newly imported cysteine-rich substrates and catalyses the formation of disulfide bounds, while the sulfhydryl oxidase
Ervl operates oxidising the reduced Mia40. ${ }^{(69)}$ Interestingly, although Ervl and cysteine-rich substrates of the MIA system are present in trypanosomatids, there is no description of a Mia40 homologue encoded by parasites' nuclear genome. ${ }^{(70,71)}$ Ervl knockdown reduces the abundance of small TIMs and cysteine-rich substrates in MIS of T. brucei, ${ }^{(72)}$ pointing to the pivotal role of this orthologue. A recent study suggests the presence of another protein that may function as a Mia40 analogue. The depletion of Mic20, a thioredoxin-like protein member of the peripheral MICOS (mitochondrial contact site and cristae organising system) subcomplex, led to a decreased import of MIS proteins, including ETS assembly factors. ${ }^{(73)}$ Other components of peripheral MICOS subcomplex also were associated with mitochondrial protein import, such as Mic32 and Mic34. ${ }^{(74)}$

Unlike yeasts and mammals, trypanosomatids have a single TIM complex that imports and inserts all proteins in mitochondrial inner membrane and matrix. TIM complex is present in a high molecular weight complex and has only the subunit Tim17 homologue to TIM22 and TIM23 found in fungi and other eukaryotes. ${ }^{(63,75)}$ It has been described that downregulation of T. brucei Tim17 by RNAi strongly inhibits the mitochondrial import of proteins and newly synthesised tRNA, ${ }^{(76)}$ suggestive of the participation in the translocation of tRNAs across the mitochondrial inner membrane. Mass spectrometry analysis identified 20 proteins associated with the parasite Tim17. Knockdown of Tim47, Tim54 and Tim62 inhibited import of mitochondrial proteins in vitro, while the disruption of Tim54 and Tim62 also negatively impacts the protein content in vivo. ${ }^{(75,77)}$ The TIM subunits Tim42 and ACAD, an orthologue of a medium chain length acyl-CoA dehydrogenase, also were described. $(54,75,77)$ It has been described two rhomboid-like proteins, named TimRhom I and TimRhom II, associated with the presequence translocase of $T$. brucei, which are essential for growth and in vivo mitochondrial protein import. $(54,55)$ The presequence translocase needs an import motor, which in yeast is composed by presequence translocase-associated motor (PAM). The orthologues of the PAM subunits: Tim44, mHsp70, Mge1, Pam18 e Pam16 were found in $T$. brucei mitochondrion. ${ }^{(78)}$ Knockdown of mHsp70 reinforced the importance of this molecule for mitochondrial import of proteins and tRNA, ${ }^{(76)}$ while the downregulation of Pam18 and Pam16 affected the growth of procyclic but not of bloodstream stage of $T$. brucei. It also has been shown that the function of Pam18 has been replaced by TbPam27, which is specifically required for the import of mitochondrial presequence. (79) A gene encoding a putative orthologue of yeast and mammal Tim50 was discovered in T. brucei It has been shown evidence that Tim50 phosphatase activity is involved in the regulation of proteins related to voltagedependent anion channel (VDAC) in parasites' mitochondrion. Since VDAC protein levels are dramatically altered according $T$. brucei life cycle stages, this data suggests that Tim50 may play a role in the regulation of mitochondrial activity. ${ }^{(80)}$ The expression of Tim50 was also described as essential for mitochondrial functionality, kDNA replication and cell cycle in bloodstream form of T. brucei. ${ }^{(81)}$ Despite that, some studies question the 


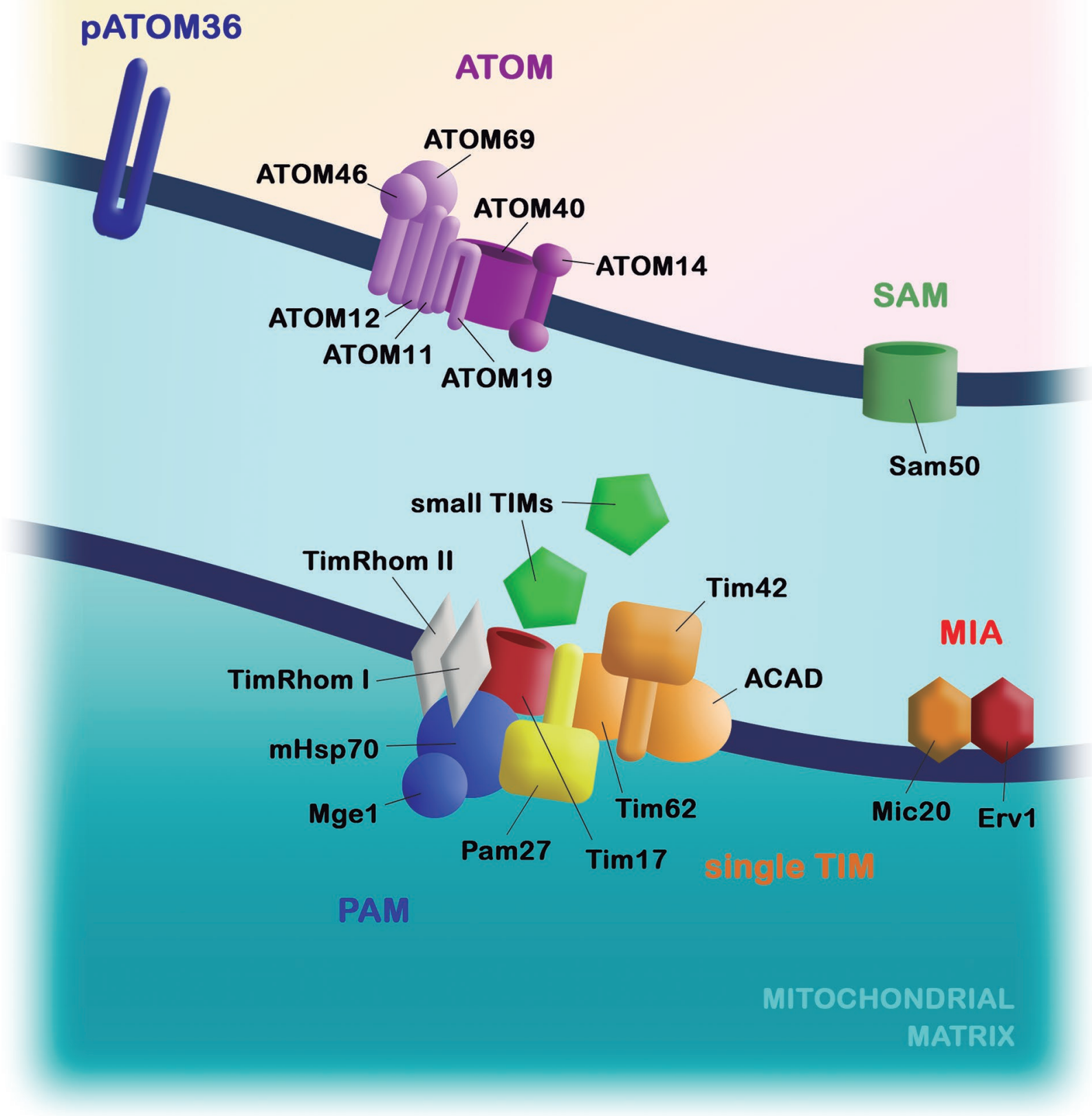

Fig. 3: mitochondrial proteins import system in Trypanosoma brucei. As in other eukaryotes, trypanosomatids present translocase of the outer membrane (TOM), sorting and assembly machinery (SAM) and translocase of the inner membrane (TIM) complexes, composed by several subunits. Many differences between mammalian and protozoan import machinery can be detected. In trypanosomatids, ATOM40, ATOM14, ATOM69, ATOM46, ATOM19, ATOM12, ATOM11, Sam 50, pATOM36 are present in the outer membrane. Small TIMs and mitochondrial intermembrane space assembly (MIA) system are found in the intermembrane space. In the inner membrane, Tim17, Tim42, Tim62, ACAD, presequence translocase-associated motor (PAM) subunits ( $\mathrm{mHsp} 70$ and Mge1) and TbPam27 are present. The presequence translocase is specifically related to TimRhom I and TimRhom II.

key role of Tim50 in these processes, since its disruption causes pleiotropic effects. Furthermore, Tim50 was not recovered in immunoprecipitations using tagged Tim subunits. ${ }^{(54,60)}$ The Fig. 3 and Table summarises all mitochondrial import machinery described in T. brucei.

\section{Concluding remarks}

Among the mitochondrial functions, oxidative phosphorylation is one of the most important. The kDNA encodes a few protein-coding genes, many of which have to be edited to become functional mRNA. Thus, the mi- 
tochondrial requirements need to be supplied by proteins encoded in nuclear genome, including enzymes involved in biosynthetic and catabolic pathways, subunits of ETS complex as well as molecules related to redox homeostase maintenance. Despite the similarities between the mitochondrial protein import system of trypanosomatids and mammals, only few subunits of their machinery are conserved. Those differences reinforce the potential of this system as a drug target, allowing the development of more efficient and safe drugs against pathogenic trypanosomatids, since there is sufficient evidence that mitochondrial protein import is essential for parasite survival and infection. Furthermore, the identification and/ or characterisation of mitochondrial import components in other pathogenic trypanosomatids are imperative.

\section{AUTHORS' CONTRIBUTION}

RFSMB conceived the work; YPR, ACSB and RFSMB drafted and wrote the manuscript. All authors approved and reviewed the manuscript.

\section{REFERENCES}

1. WHO - World Health Organization. Chagas disease (American trypanosomiasis). Fact Sheet. 2020. Available from: https//www. who.int/news-room/fact-sheets/detail/chagas-disease-(americantrypanosomiasis).

2. Field MC, Horn D, Fairlamb AH, Ferguson MAJ, Gray DW, Read $\mathrm{KD}$, et al. Anti-trypanosomatid drug discovery: an ongoing challenge and a continuing need. Nat Rev Microbiol. 2017; 15(4): 217-31.

3. Nussbaum K, Honek J, Cadmus C, Efferth T. Trypanosomatid parasites causing neglected diseases. Curr Med Chem. 2010; 17: 1594-617.

4. de Souza W, Attias M, Rodrigues JCF. Particularities of mitochondrial structure in parasitic protists (Apicomplexa and Kinetoplastida). Int J Biochem Cell Biol. 2009; 41: 2069-80.

5. Fidalgo LM, Gille L. Mitochondria and trypanosomatids: targets and drugs. Pharm Res. 2011; 28: 2758-70.

6. Menna-Barreto RFS, Goncalves RLS, Costa EM, Silva RSF, Pinto AV, Oliveira MF, et al. The effects on Trypanosoma cruzi of novel synthetic naphthoquinones are mediated by mitochondrial dysfunction. Free Radic Biol Med. 2009; 47: 644-53.

7. Luque-Ortega JR, Reuther P, Rivas L, Dardonville C. New Benzophenone-derived bisphosphonium salts as leishmanicidal leads targeting mitochondria through inhibition of respiratory complex II. J Med Chem. 2010; 53(4): 1788-98.

8. Menna-Barreto RFS, De Castro SL. The double-edged sword in pathogenic trypanosomatids: the pivotal role of mitochondria in oxidative stress and bioenergetics. Biomed Res Int. 2014; 2014: 614014.

9. Vannier-Santos MA, Brunoro GF, Soeiro MNC, DeCastro SL, Menna-Barreto R. Parasite, compartments and molecules: trick vs treatment on Chagas disease. In: de Souza W, editor. Biology of Trypanosoma cruzi. Rijeka: IntechOpen; 2019.

10. Menzies SK, Tulloch LB, Florence GJ, Smith TK. The trypanosome alternative oxidase: a potential drug target? Parasitology. 2018; 145: 175-83.

11. Opperdoes FR, Borst P, Bakker S, Leene W. Localization of glycerol-3-phosphate oxidase in the mitochondrion and particulate NAD+-linked glycerol-3-phosphate dehydrogenase in the microbodies of the bloodstream form to Trypanosoma brucei. Eur J Biochem. 1977; 76(1): 29-39.
12. Urbaniak MD, Guther MLS, Ferguson MAJ. Comparative SILAC proteomic analysis of Trypanosoma brucei bloodstream and procyclic lifecycle stages. PLoS One. 2012; 7(5): e36619.

13. Zíková A, Verner Z, Nenarokova A, Michels PAM, Lukeš J. A paradigm shift: the mitoproteomes of procyclic and bloodstream Trypanosoma brucei are comparably complex. PLoS Pathog. 2017; 13(12): e1006679.

14. Santhamma KR, Bhaduri A. Characterization of the respiratory chain of Leishmania donovani promastigotes. Mol Biochem Parasitol. 1995; 75: 43-53.

15. Chaudhuri M, Ott RD, Hill GC. Trypanosome alternative oxidase: from molecule to function. Trends Parasitol. 2006; 22(10): 484-91.

16. Venditti P, Di Stefano L, Di Meo S. Mitochondrial metabolism of reactive oxygen species. Mitochondrion. 2013; 13: 71-82.

17. Opperdoes FR, Michels PAM. Complex I of Trypanosomatidae: does it exist? Trends Parasitol. 2008; 24: 310-7.

18. Carranza JC, Kowaltowski AJ, Mendonça MAG, De Oliveira TC, Gadelha FR, Zingales B. Mitochondrial bioenergetics and redox state are unaltered in Trypanosoma cruzi isolates with compromised mitochondrial complex I subunit genes. J Bioenerg Biomembr. 2009; 41: 299-308.

19. Tomás AM, Castro H. Redox metabolism in mitochondria of trypanosomatids. Antioxid Redox Signal. 2013; 19: 696-707.

20. Vercesi AE, Bernardes CF, Hoffmann ME, Gadelha FR, Docampo R. Digitonin permeabilization does not affect mitochondrial function and allows the determination of the mitochondrial membrane potential of Trypanosoma cruzi in situ. J Biol Chem. 1991; 266: 14431-4.

21. Mehta A, Shaha C. Apoptotic death in Leishmania donovani promastigotes in response to respiratory chain inhibition: complex II inhibition results in increased pentamidine cytotoxicity. J Biol Chem. 2004; 279: 11798-813.

22. Genes C, Baquero E, Echeverri F, Maya JD, Triana O. Mitochondrial dysfunction in Trypanosoma cruzi: the role of Serratia marcescens prodigiosin in the alternative treatment of Chagas disease. Parasit Vectors. 2011; 4: 66.

23. Gonçalves RLS, Menna-Barreto RFS, Polycarpo CR, Gadelha FR, Castro SL, Oliveira MF. A comparative assessment of mitochondrial function in epimastigotes and bloodstream trypomastigotes of Trypanosoma cruzi. J Bioenerg Biomembr. 2011; 43(6): 651-61.

24. Murphy MP. How mitochondria produce reactive oxygen species. Biochem J. 2009; 417: 1-13.

25. Wang Y, Hekimi S. Understanding ubiquinone. Trends Cell Biol. 2016; 26: 367-78.

26. Ray PD, Huang BW, Tsuji Y. Reactive oxygen species (ROS) homeostasis and redox regulation in cellular signaling. Cell Signal. 2012; 24: 981-90.

27. Sies H, Berndt C, Jones DP. Oxidative stress. Annu Rev Biochem. 2017; 86: 715-48.

28. Powis G. Metabolism and reactions of quinoid anticancer agents. Pharmacol Ther. 1987; 35(1-2): 57-162.

29. O’Brien PJ. Molecular mechanisms of quinone cytotoxicity. Chem Biol Interact. 1991; 80: 1-41.

30. Carvalho L, Luque-Ortega JR, Manzano JI, Castanys S, Rivas L, Gamarro F. Tafenoquine, an antiplasmodial 8-aminoquinoline, targets Leishmania respiratory complex III and induces apoptosis. Antimicrob Agents Chemother. 2010; 54(12): 5344-51.

31. Kathuria M, Bhattacharjee A, Sashidhara KV, Singh SP, Mitra $\mathrm{K}$. Induction of mitochondrial dysfunction and oxidative stress in Leishmania donovani by orally active clerodane diterpene. Antimicrob Agents Chemother. 2014; 58: 5916-28. 
32. Bombaça ACS, Viana PG, Santos ACC, Silva TL, Rodrigues ABM, Guimarães ACR, et al. Mitochondrial disfunction and ROS production are essential for anti-Trypanosoma cruzi activity of $\beta$-lapachone-derived naphthoimidazoles. Free Radic Biol Med. 2019; 130: 408-18.

33. Bombaça ACS, Silva LA, Chaves OA, da Silva LS, Barbosa JMC, da Silva AM, et al. Novel N,N-di-alkylnaphthoimidazolium derivative of $\boldsymbol{\beta}$-lapachone impaired Trypanosoma cruzi mitochondrial electron transport system. Biomed Pharmacother. 2021; 135: 111186.

34. Boveris A, Docampo R, Turrens JF, Stoppani AOM. Effect of beta-lapachone on superoxide anion and hydrogen peroxide production in Trypanosoma cruzi. Biochem J. 1978; 175: 431-9.

35. Docampo R, Lopes JN, Cruz FS, de Souza W. Trypanosoma cruzi: ultrastructural and metabolic alterations of epimastigotes by betalapachone. Exp Parasitol.1977; 42: 142-9.

36. Docampo R, Cruz FS, Boveris A, Muniz RPA, Esquivel DMS. Lipid peroxidation and the generation of free radicals, superoxide anion, and hydrogen peroxide in beta-lapachone-treated Trypanosoma cruzi epimastigotes. Arch Biochem Biophys. 1978; 186: 292-7.

37. Pinto AV, Pinto CN, Pinto MC, Rita RS, Pezzella CA, de Castro SL. Trypanocidal activity of synthetic heterocyclic derivatives of active quinones from Tabebuia sp. Arzneimittelforschung. 1997; 47(1): 74-9.

38. Pinto CN, Dantas AP, De Moura KCG, Emery FS, Polequevitch PF, Pinto MCFR, et al. Chemical reactivity studies with naphthoquinones from Tabebuia with anti-trypanosomal efficacy. Arzneimittelforschung. 2000; 50: 1120-8.

39. De Moura KCG, Salomão K, Menna-Barreto RFS, Emery FS, Pinto MDCFR, Pinto AV, et al. Studies on the trypanocidal activity of semi-synthetic pyran[b-4,3]naphtho[1,2-d]imidazoles from betalapachone. Eur J Med Chem. 2004; 39: 639-45.

40. Menna-Barreto RFS, Henriques-Pons A, Pinto AV, Morgado-Diaz JA, Soares MJ, De Castro SL. Effect of a beta-lapachone-derived naphthoimidazole on Trypanosoma cruzi: identification of target organelles. J Antimicrob Chemother. 2005; 56: 1034-41.

41. Menna-Barreto RFS, Corrêa JR, Pinto AV, Soares MJ, De Castro SL. Mitochondrial disruption and DNA fragmentation in Trypanosoma cruzi induced by naphthoimidazoles synthesized from $\beta$-lapachone. Parasitol Res. 2007; 101: 895-905.

42. Garavaglia PA, Cannata JJB, Ruiz AM, Maugeri D, Duran R, Galleano M, et al. Identification, cloning and characterization of an aldo-keto reductase from Trypanosoma cruzi with quinone oxidoreductase activity. Mol Biochem Parasitol. 2010; 173: 132-41.

43. Dantas-Pereira L, Cunha-Junior EF, Andrade-Neto VV, Bower JF, Jardim GAM, da Silva Jr EN, et al. Naphthoquinones and derivatives for chemotherapy: perspectives and limitations of their antitrypanosomatids activities. Curr Pharm Des. 2021; 27: 1807-24.

44. Mehlotra RK. Antioxidant defense mechanisms in parasitic protozoa. Crit Rev Microbiol. 1996; 22: 295-314.

45. Flohé L, Hecht HJ, Steinert P. Glutathione and trypanothione in parasitic hydroperoxide metabolism. Free Radic Biol Med. 1999; 27: 966-84.

46. Fairlamb AH, Blackburn P, Ulrich P, Chait BT, Cerami A. Trypanothione: a novel bis(glutathionyl)spermidine cofactor for glutathione reductase in trypanosomatids. Science. 1985; 227: 1485-7.

47. Krauth-Siegel RL, Comini MA. Redox control in trypanosomatids, parasitic protozoa with trypanothione-based thiol metabolism. Biochim Biophys Acta. 2008; 1780: 1236-48.

48. Manta B, Comini M, Medeiros A, Hugo M, Trujillo M, Radi R. Trypanothione: a unique bis-glutathionyl derivative in trypanosomatids. Biochim Biophys Acta. 2013; 1830: 3199-216.
49. Leroux AE, Krauth-Siegel RL. Thiol redox biology of trypanosomatids and potential targets for chemotherapy. Mol Biochem Parasitol. 2016; 206: 67-74.

50. Krieger S, Schwarz W, Arlyanayagam MR, Fairlamb AH, KrauthSiegel RL, Clayton C. Trypanosomes lacking trypanothione reductase are avirulent and show increased sensitivity to oxidative stress. Mol Microbiol. 2000; 35: 542-52.

51. Torrie LS, Wyllie S, Spinks D, Oza SL, Thompson S, Harrison JR, et al. Chemical validation of trypanothione synthetase: a potential drug target for human trypanosomiasis. J Biol Chem. 2009; 284(52): 36137-45.

52. Pusnik M, Schmidt O, Perry AJ, Oeljeklaus S, Niemann M, Warscheid B, et al. Mitochondrial preprotein translocase of trypanosomatids has a bacterial origin. Curr Biol. 2011; 21: 1738-43.

53. Mani J, Desy S, Niemann M, Chanfon A, Oeljeklaus S, Pusnik $\mathrm{M}$, et al. Mitochondrial protein import receptors in Kinetoplastids reveal convergent evolution over large phylogenetic distances. Nat Commun. 2015; 6: 6646.

54. Harsman A, Oeljeklaus S, Wenger C, Huot JL, Warscheid B, Schneider A. The non-canonical mitochondrial inner membrane presequence translocase of trypanosomatids contains two essential rhomboid-like proteins. Nat Commun. 2016; 7: 13707.

55. Harsman A, Schneider A. Mitochondrial protein import in trypanosomes: expect the unexpected. Traffic. 2017; 18: 96-109.

56. Sharma S, Singha UK, Chaudhuri M. Role of Tob55 on mitochondrial protein biogenesis in Trypanosoma brucei. Mol Biochem Parasitol. 2010; 174: 89-100.

57. Niemann M, Wiese S, Mani J, Chanfon A, Jackson C, Meisinger $\mathrm{C}$, et al. Mitochondrial outer membrane proteome of Trypanosoma brucei reveals novel factors required to maintain mitochondrial morphology. Mol Cell Proteomics. 2013; 12: 515-28.

58. Pusnik M, Mani J, Schmidt O, Niemann M, Oeljeklaus S, Schnarwiler $\mathrm{F}$, et al. An essential novel component of the noncanonical mitochondrial outer membrane protein import system of trypanosomatids. Mol Biol Cell. 2012; 23: 3420-8.

59. Käser S, Willemin M, Schnarwiler F, Schimanski B, PovedaHuertes D, Oeljeklaus S, et al. Biogenesis of the mitochondrial DNA inheritance machinery in the mitochondrial outer membrane of Trypanosoma brucei. PLoS Pathog. 2017; 13: e1006808.

60. Schneider A. Evolution of mitochondrial protein import - lessons from trypanosomes. Biol Chem. 2020; 401: 663-76.

61. Vitali DG, Käser S, Kolb A, Dimmer KS, Schneider A, Rapaport D. Independent evolution of functionally exchangeable mitochondrial outer membrane import complexes. 2018; 7: e34488.

62. Käser S, Oeljeklaus S, Týc J, Vaughan S, Warscheid B, Schneider A. Outer membrane protein functions as integrator of protein import and DNA inheritance in mitochondria. Proc Natl Acad Sci USA. 2016; 113: E4467-75.

63. Schneider A, Ochsenreiter T. Failure is not an option - mitochondrial genome segregation in trypanosomes. J Cell Sci. 2018; 131(18): jcs221820.

64. Gentle IE, Perry AJ, Alcock FH, Likić VA, Dolezal P, Ng ET, et al. Conserved motifs reveal details of ancestry and structure in the small TIM chaperones of the mitochondrial intermembrane space. Mol Biol Evol. 2007; 24: 1149-60.

65. Chaudhuri M, Darden C, Gonzalez FS, Singha UK, Quinones L, Tripathi A. Tim17 updates: a comprehensive review of an ancient mitochondrial protein translocator. Biomolecules. 2020; 10: 1-20.

66. Eckers E, Cyrklaff M, Simpson L, Deponte M. Mitochondrial protein import pathways are functionally conserved among eukaryotes despite compositional diversity of the import machineries. Biol Chem. 2012; 393: 513-24. 
67. Wenger C, Oeljeklaus S, Warscheid B, Schneider A, Harsman A. A trypanosomal orthologue of an intermembrane space chaperone has a non-canonical function in biogenesis of the single mitochondrial inner membrane protein translocase. PLoS Pathog. 2017; 13(8): e1006550.

68. Smith JT, Singha UK, Misra S, Chaudhuri M. Divergent Small Tim homologues are associated with TbTim17 and critical for the biogenesis of TbTim 17 protein complexes in Trypanosoma brucei. mSphere. 2018; 3(3): e00204-18.

69. Allen JWA, Ferguson SJ, Ginger ML. Distinctive biochemistry in the trypanosome mitochondrial intermembrane space suggests a model for stepwise evolution of the MIA pathway for import of cysteine-rich proteins. FEBS Lett. 2008; 582: 2817-25.

70. Basu S, Leonard JC, Desai N, Mavridou DAI, Tang KH, Goddard $\mathrm{AD}$, et al. Divergence of Erv1-associated mitochondrial import and export pathways in trypanosomes and anaerobic protists. Eukaryot Cell. 2013; 12: 343-55.

71. Eckers E, Petrungaro C, Gross D, Riemer J, Hell K, Deponte M. Divergent molecular evolution of the mitochondrial sulfhydryl:cytochrome C oxidoreductase Erv in opisthokonts and parasitic protists. J Biol Chem. 2013; 288: 2676-88.

72. Peikert CD, Mani J, Morgenstern M, Käser S, Knapp B, Wenger $\mathrm{C}$, et al. Charting organellar importomes by quantitative mass spectrometry. Nat Commun. 2017; 8: 15272.

73. Kaurov I, Vancová M, Schimanski B, Cadena LR, Heller J, Bílý $\mathrm{T}$, et al. The diverged Trypanosome MICOS Complex as a hub for mitochondrial cristae shaping and protein import. Curr Biol. 2018; 28: 3393-3407.e5.

74. Eichenberger C, Oeljeklaus S, Bruggisser J, Mani J, Haenni B, Kaurov I, et al. The highly diverged trypanosomal MICOS complex is organized in a nonessential integral membrane and an essential peripheral module. Mol Microbiol. 2019; 112: 1731-43.

75. Singha UK, Hamilton VN, Duncan MR, Weems E, Tripathi MK, Chaudhuri M. Protein translocase of mitochondrial inner membrane in Trypanosoma brucei. J Biol Chem. 2012; 287: 14480-93.

76. Tschopp F, Charrière F, Schneider A. In vivo study in Trypanosoma brucei links mitochondrial transfer RNA import to mitochondrial protein import. EMBO Rep. 2011; 12: 825-32.

77. Singha UK, Hamilton V, Chaudhuri M. Tim62, a novel mitochondrial protein in Trypanosoma brucei, is essential for assembly and stability of the TbTim 17 protein complex. J Biol Chem. 2015; 290: 23226-39.
78. Fukasawa Y, Oda T, Tomii K, Imai K. Origin and evolutionary alteration of the mitochondrial import system in eukaryotic lineages. Mol Biol Evol. 2017; 34: 1574-86.

79. Känel C, Muñoz-Gómez SA, Oeljeklaus S, Wenger C, Warscheid B, Wideman JG. Homologue replacement in the import motor of the mitochondrial inner membrane of trypanosomes. Elife. 2020; 9: e52560.

80. Duncan MR, Fullerton M, Chaudhuri M. Tim50 in Trypanosoma brucei possesses a dual specificity phosphatase activity and is critical for mitochondrial protein import. J Biol Chem. 2013; 288: $3184-97$

81. Tripathi A, Singha UK, Cooley A, Gillyard T, Krystofiak E, Pratap S, et al. Trypanosoma brucei Tim50 possesses PAP activity and plays a critical role in cell cycle regulation and parasite infectivity. MBio. 2021; 12(5): e0159221.

82. Harsman A, Niemann M, Pusnik M, Schmidt O, Burmann BM, Hiller S, et al. Bacterial origin of a mitochondrial outer membrane protein translocase: new perspectives from comparative single channel electrophysiology. J Biol Chem. 2012; 287: 31437-45.

83. Niemann M, Harsman A, Mani J, Peikert CD, Oeljeklaus S, Warscheid $B$, et al. tRNAs and proteins use the same import channel for translocation across the mitochondrial outer membrane of trypanosomes. Proc Natl Acad Sci USA. 2017; 114: E7679-87.

84. Povelones ML, Tiengwe C, Gluenz E, Gull K, Englund PT, Jensen RE. Mitochondrial shape and function in trypanosomes requires the outer membrane protein, TbLOK1. Mol Microbiol. 2013; 87: 713-29.

85. Desy S, Mani J, Harsman A, Käser S, Schneider A. TbLOK1/ ATOM19 is a novel subunit of the noncanonical mitochondrial outer membrane protein translocase of Trypanosoma brucei. Mol Microbiol. 2016; 102: 520-9.

86. Singha UK, Peprah E, Williams S, Walker R, Saha L, Chaudhuri $\mathrm{M}$. Characterization of the mitochondrial inner membrane protein translocator Tim 17 from Trypanosoma brucei. Mol Biochem Parasitol. 2008; 159: 30-43.

87. Shikha S, Huot JL, Schneider A, Niemann M. tRNA import across the mitochondrial inner membrane in T. brucei requires TIM subunits but is independent of protein import. Nucleic Acids Res. 2020; 48: 12269-81.

88. Týč J, Klingbeil MM, Lukeš J. Mitochondrial heat shock protein machinery hsp70/hsp40 is indispensable for proper mitochondrial DNA maintenance and replication. MBio. 2015; 6(1): e02425-14. 